\author{
Guilherme Machado de Carvalho*, \\ Vanessa Gonçalves Dias, Hugo Kohler, \\ Carlos Takahiro Chone, Alexandre \\ Caixeta Guimarães, Agrício Nubiato \\ Crespo \\ Department of Ear, Nose, Throat and Head and \\ Neck Surgery, Campinas University, Sao Paulo, \\ Brazil \\ Dates: Received: 12 July, 2016; Accepted: 04 \\ August, 2016; Published: 05 August, 2016 \\ *Corresponding author: Professor, Guilherme \\ Carvalho, Ear, Nose, Throat and Head and Neck \\ Surgery Department, Campinas University, UNICAMP, \\ PO BOX 6111, Postal Code: 13081-970, Sao Paulo, \\ Brazil, Tel: +55 19 35217523; Fax: +55 1935217563 ; \\ E-mail: guimachadocarvalho@gmail.com \\ www.peertechz.com \\ ISSN: 2455-4634
}

Keywords: Sentinel lymph node biopsy; Lymphatic metastasis; Squamous cell carcinoma; Oral cavity; Neck dissection

\section{Research Article \\ Sentinel Lymph Node Biopsy vs. Elective Neck Dissection in Patients with T1/T2 N0 Oral Squamous Cell Carcinoma: A Matched Pair Analysis}

\begin{abstract}
Background: The management of the clinically and radiological negative neck in patients with early squamous cell carcinoma of head and neck (HNSCC) is still controversial. As approximately 20 to $30 \%$ of these patients harbor occult neck disease, most of them is submitted to elective neck dissection with no benefit in great majority of those cases. Sentinel lymph node biopsy (SLB) is a potential method for staging of lymphatic metastasis in HNSCC and the status of the sentinel node predicts the presence of metastasis in the remainder of the nodes within the nodal basin.

Objective: To evaluate the neck recurrence rate of patients with early stage (T1/T2) HNSCC of the oral cavity with clinically negative necks (cNO), in patients submitted to sentinel lymph node biopsy (SNB) or elective neck dissection (END).

Methods: Clinical retrospective comparison of two patient cohorts of early stage oral cancer tested for primary tumor resection and SNB without subsequent END or primary tumor resection with END. Those comparisons were made by a matched pair analysis.

Results: The current study included 52 patients with early stage oral cancer; 30 in the SNB group and 22 in the control group (END). Both SNB and END groups had similar performance regarding the recurrence rate of neck, without significant difference between both groups. Conclusion: Neck recurrence rates were similar between groups of patients in which SNB or END was performed.
\end{abstract}

\section{Introduction}

Squamous cell carcinoma of the head and neck (HNSCC) spreads regionally to the lymphatic system [1]. An important prognostic factor for head and neck cancer is the lymph node metastasis, which may reduce five-year survival rate by $50 \%$ [2]. However, controversy still remains regarding the optimal techniques to determine if patients with a clinically negative neck (cN0) actually have lymph node metastases.

Clinical examination and radiological techniques are associated with approximately $30 \%$ false negative and positive rates for identification of lymph node metastases [3-8]. Histopathological examination of the surgical specimen following neck dissection is considered the gold standard method for neck staging [9].

Neck dissection must be considered in patients whose primary lesion has an expected rate of lymphatic metastases of more than $20 \%$ $[1,2]$. Even patients with early HNSCC of oral cavity (OC), more than two millimeters of depth suggests considerable risk of metastasis and elective neck dissection (END) must be performed [10-12].

Neck dissection in addition to resection of the primary tumor may lead to increased surgical length and morbidity, an this is why it is important to study and develop alternative ways of accurately staging the clinically negative (cN0) neck. Meanwhile, END still is the gold standard approach for staging the cervical lymph nodes in patients with HNSCC.

END is simultaneously a therapeutic and staging procedure that may be beneficial for patients who are subsequently found to harbor occult disease. However, these situations occurs in approximately $25 \%$ of those cases, leading to the possibility of $75 \%$ of the remains patients are being over treated $[13,14]$.

There was a prospective randomized study in the literature to compare elective ipsilateral radical neck dissection versus observation in the treatment of stage I to II oral tongue carcinoma in India [15]. Another prospective randomized study of T1-3N0M0 of mixed oral tongue and floor of mouth carcinomas was conducted in France comparing observation and elective ipsilateral radical neck dissection [16]. These 2 prospective randomized studies did not show any survival benefit of elective radical neck dissection, but it has to be noted that those papers had a limitations regarding to the number of patients included.

Another prospective randomized study of selective neck dissection (levels I, II, III) versus observation for N0 neck of stage I to II oral tongue carcinoma shows that the 5-year disease-specific survival rate was $87 \%$ for the observation group and was $89 \%$ for the END group; the $2 \%$ difference was not significant [16]. 
In recent decades, sentinel lymph node biopsy (SNB) has been employed for staging and treatment of oral cancer in patients with cN0 necks. The ability to limit the pathological evaluation to a small number of nodes potentially offers an additional benefit: It is possible to use more detailed pathological techniques to identify micrometastatic deposits, a process that would be impractical for a complete neck dissection specimen [13]

The SNB technique has a high rate of sensitivity and high negative and positive predictive values in early stage (T1 and T2) oral cancer with $\mathrm{cN} 0$ and radiological negative necks [17-19]. Thus, the identification and excision of sentinel lymph node, even when histopathologically positive for metastases, appears to be safe [12,21].

In cohorts where subsequent END was performed after SNB, only 3 to $5 \%$ of additional metastatic lymph nodes in the surgical specimen of END were harvested, suggesting a low probability of neck recurrence after SNB without neck dissection when the sentinel node is histopathologically negative $[13,21,22]$.

However, it does not follow that the recurrence rate will be zero only by negative histological evaluation. The detection and histological evaluation method still has technical limitations and this is one of the reasons that the disease recurrence still occurs. Therefore this is a limitation on the argument that the recurrence rate in the dissected lymph nodes could be less than those that exist in the removed lymph nodes, although, this can be only an assumption theory.

Paradoxically, some medical literature articles support that this recurrence rate in the dissected lymph nodes would actually be lower, based on data presented by their respective evaluations, reported in the literature following supraomohyoid neck dissection (of up to 10\%), and even after radical neck dissection in cN0 and histopathologically negative necks $(6.7 \%)[23,24]$. These situations lead us to identify the consensus lack and scientific medical discussion that are still around this issue.

The aim of current study was to compare the neck recurrence rate for two treatment modalities (END or SNB, plus excision of local tumor) for HNSCC oral cavity patients with early stage (T1/T2 N0).

\section{Patient and Methods}

A retrospective study with patients with HNSCC (squamous cell carcinoma) of the oral cavity, confirmed by histopathological examination at our institution was developed. The primary lesion in these patients was staged as T1 or T2 according to the AJCC 2010, and treated with SNB or END plus excision of the local tumor [25].

All $\mathrm{cNO}$ and radiologically negative necks were evaluated by multi-slice CT scan with 128 detectors. Patients submitted to SNB were prospectively followed without subsequent neck dissection.

Adjuvant treatment (radiotherapy and induced chemotherapy) was indicated in patients with positive margins, extra capsular spread, presence of perineural invasion, or vascular emboli. All patients were followed postoperatively with a CT scan every six months. All patients who did not complete these criteria were excluded from the study.

\section{Study group - SNLB technique}

SLNB was performed via peritumoral injection of technetium. Lymphoscintigraphy and SPECT-CT scan (Single-photon emission computed tomography) was performed in all cases. The skin of neck was marked accordingly and a gamma probe was used for identification of sentinel lymph node. Step serial sections of the sentinel lymph node were stained with hematoxylin-eosin and immunohistochemistry was performed. This was an option to increase the diagnostic accuracy but not to decrease the recurrence rate.

\section{Control group}

The control group submitted to END was retrospectively collected via review of medical charts of patients with T1/T2 oral cancer, cN0 and radiologically negative necks. This group was match-paired by age, gender, stage, site, histopathological status of primary lesion and neck dissection specimen, adjuvant treatment, and length of followup.

\section{Statistical analysis}

The data were analyzed using descriptive analysis, with production of means, medians, standard deviation tabs.

Chi-Square was used to compare the groups of our sample. Because of the small size of some of the variables analyzed Fisher's exact test was also used to check the correlation between the groups, and the Odds-Ratio (OR) was also calculated. The OR was used because our selected sample for this study was almost our wholly population for this matter.

The confidence interval was of $95 \%$, and p-value $<0.05$ was considered significant.

\section{Ethical considerations}

This study was previously approved by the Research Ethics Committee of the Faculty of Medical Sciences of the University of Campinas (Report number 396/2006).

\section{Results}

The current study selected 68 patients, but included only 52 patients because of the inclusion criteria, 30 in the SNB group (8 excluded), and 22 in the control group (END) (8 excluded). There was a preponderance of men $(83 \%)$, with a similar distribution between the groups $(\mathrm{OR}=1.11$, Table 1$)$. The tumor $\mathrm{T}$ stage distribution of patients in each group was similar $(\mathrm{OR}=0.96)$ as shown in Table 1.

The average age of patients in the SLN group was 58.86 years and in the control group was 58.1 years; there was no significant difference between groups.

Lymph node metastases occurred in seven (23\%) patients in the SNB group, and in seven (32\%) patients in the control group $(\mathrm{OR}=0.53$; Table 1). In the SNB group, all identified metastases were in the dissected sentinel lymph node. It has to be noted that there was no elective neck dissection and not all lymph nodes were available and analyzed histologically for metastasis.

Vascular emboli determined via histopathological analysis was present in 6 patients $(27 \%)$ in the SLN group and 7 patients in the END (23\%) group ( $\mathrm{OR}=1.23$, without association).

Recurrence was observed in two subjects at SNB (6,6\%) group and one at END (4,5\%) group, respectively. Recurrences was observed 
with a meantime of 8,6 months in SNB group and 9,4 months in END group.

All recurrences were treated with radical neck dissection. In $78 \%$ of positive SNB cases and $85 \%$ of positive cases in END group, postoperative radiation therapy was required due to presence of perineural invasion, vascular emboli or extra capsular spread in lymphatic metastases. Both the SNB and the END groups had similar rates of neck recurrence, with no statistical difference between then $(\mathrm{p}=0,68)$.

As shown in Figure 1, both the SLNB and the END groups had similar rates of neck recurrence, with no statistical difference between the groups $(\mathrm{p}=0,68)$.

Figure 1 illustrates that the SLNB and END subjects had similar performance as the recurrence of cervical metastases, with no statistical difference between the groups $((\mathrm{p}=0,68)$, demonstrating that the END group only increased surgical comorbidities and risk involved with the procedure.

\begin{tabular}{|c|c|c|}
\hline & END & SLNB \\
\hline \multicolumn{3}{|l|}{ Gender } \\
\hline male & 18 & 25 \\
\hline female & 4 & 5 \\
\hline \multicolumn{3}{|l|}{ Stage } \\
\hline pT1 & 6 & 8 \\
\hline pT2 & 16 & 22 \\
\hline \multicolumn{3}{|c|}{ Status of neck } \\
\hline Meta (-) & 15 & 23 \\
\hline Meta $(+)$ & 7 & 7 \\
\hline Total & 22 & 30 \\
\hline
\end{tabular}

END, elective neck dissection; SLNB, sentinel lymph node biopsy; pT1 and pT2, histopathological stage of primary tumor; Meta (-), abscence of lymph node metastases; Meta (+), presence of lymph node metastases.

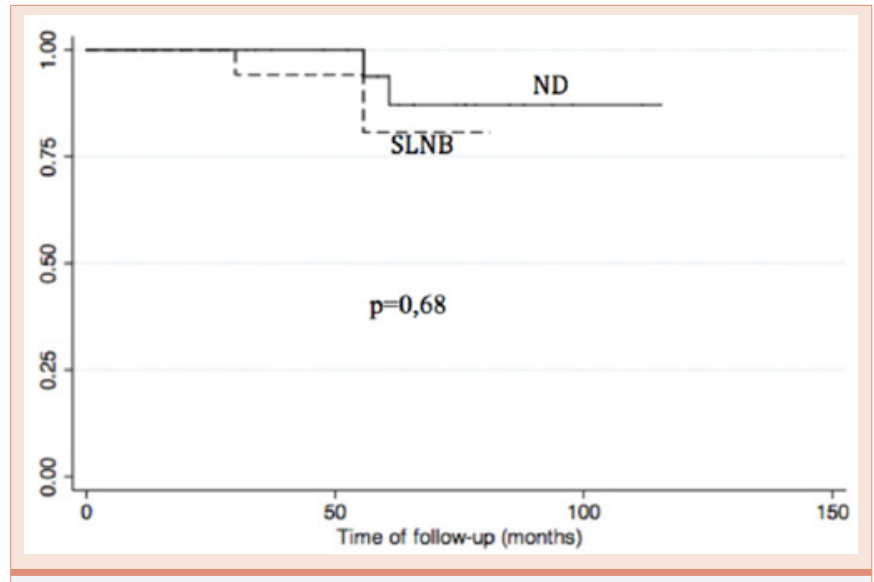

Figure 1: Figure shows the layout of the survival analysis of groups of SNLB and END.

$\mathrm{ND}=\mathrm{EN}$ D= elective neck dissection; SNLB =sentinel lymph node biopsy.

\section{Discussion}

SNB consensus for HNSCC is slower than that for melanoma; however, initial results have demonstrated being a good treatment option and also a staging method for those patients. Early phase I validation studies have consistently reported good sensitivity and negative predictive values of greater than $90 \%$, stimulation for a larger multicenter trials such as 5-year follow-up of a European multicenter trial $[14,26]$.

SNB technique has been extensively debated in the literature for HNSCC of the oral cavity. Between 1996 and 2000, several institutions conducted studies on this subject. Over 60 trials were conducted, along with two international conferences, a meta-analysis, and recent descriptions regarding SNB practices was published [19,27].

In this context, the literature suggests that the negative predictive value of SNB ranges from 90 to $100 \%$ for early stage oral cancer, and immunohistochemistry is essential for proper SNB evaluation [28]. Thus, Sentinel node biopsy is a reliable and reproducible means of staging the clinically N0 neck for patients with cT1/T2 HNSCC 14.

SNB avoids unnecessary neck dissection in patients with early stage HNSCC of oral cavity with negative sentinel lymph node which suggests very low risk of occult lymphatic metastases in the remaining lymphatic drainage (3 to $5 \%$ ). The prevailing view regarding END is that neck dissection should be performed in a patient with a cN0 only if the risk of occult metastasis is greater than 20\% [27-29].

The negative predictive value of SNB was extensively studied through a prospective, multicenter study, with value of $96 \%$ in patients with T1 and T2 HNSCC of oral cavity, and of $100 \%$ for T1 lesions [31]. A systematic review with meta-analysis also observed excellent safety and good sensitivity in identifying occult lymphatic metastases in cN0 neck of early stage oral cancer patients [19].

The results of phase I trials have proven encouraging, with several small single center studies reporting technical success rates, sensitivities, and negative predictive values greater than 90\% [26,31]. While these results suggest the feasibility of SNB, larger phase II and III trials are required before the technique can be recommended as a true alternative to END in this population.

To date, the European multicenter trial provides the most compelling evidence to support the use of SNB as a staging tool [14]. Furthermore, in a prospective study comparing postoperative complications and quality of life in patients with HNSCC of the oral cavity and oropharynx submitted to either SNB or END, fewer complications and improved quality of life was observed in those patients who underwent SNB [30].

A European multicenter trial compared loco regional diseasefree survival between SNB-positive and SNB-negative patient groups. This study had a 5 years of follow-up. The presented data in this big trial appears to demonstrate poorer survival rate in the SNBpositive group, but this finding did not reach significance statistically (log-rank statistic 0.37, $p=0.55$ ). Comparison between SNB alone and SNB-assisted with END demonstrated no significant survival difference for patients with malignant tumors the mouth [14]. 
Studies regarding SNB are critical to avoid neck dissection procedures and their associated morbidities, such as shoulder dysfunction, lower lip paresis and extensive scarring of the neck and to improve postoperative quality of life of these patients. Inspite of the high rate of sensitivity and negative predictive value of SNB in oral cancer, there are no studies comparing the rate of neck recurrence between SNB and END in a comparative analysis. Like the European multicenter trial ${ }^{14}$, perhaps most importantly, patients undergoing sentinel node biopsy alone were not demonstrated to have a significantly different long-term survival compared with patients undergoing elective neck dissection in this study [14].

Thus, our study observed that both the SNB and END groups had similar neck recurrence rates, without statistical differences between them. However, its exact role in the management of these patients remains largely undefined. At present, there are 2 ongoing multicenter trials whose outcomes may prove of considerable importance: the European Sentinel Node Trial (SENT) and The American College of Surgeons Oncology Group (ACOSOG) Z0360 trials [32,33].

The rate of neck recurrence after SNB without neck dissection was not statistically significant compared to END in early stage HNSCC of the oral cavity in a comparative analysis. However, evidence favoring its use as a staging tool continues to grow, and the results of this study agree with other studies. The postoperative follow-up in the described analysis above was short, but already has shown that the recurrence of the disease. The authors believe that a longer follow up time would bring more reliable conclusions.

The authors believe that this retrospective data will not solve the problem but is good attempt to address it and gives preliminary information. Another study bias is that is that most patients, almost $80 \%$, in both groups were treated with post-operative radiation. Given the previous N0 status, that radiation to the neck alone could have being an extra fator of treating the neck without any surgery [34].

The patient follow up will be crucial for understanding other findings and data os this study. The fact that only $3-5 \%$ of non-sentinel nodes are positive at the time of examination does not mean that this would be the recurrence rate in the future because some nodes will harbor metastases that can only be detected with molecular methods such as PCR. Other nodes will harbor metastases that cannot be identified by all means. Any diagnostic test will have a false-negative rate. Some disease will only appear with follow-up over time. That is why relapse happens in both $\mathrm{p}$-ve and $\mathrm{p}+\mathrm{ve}$ cases.

While SNB may not be universally applicable in this patient population, its potential benefits are clear and the upcoming results of ongoing multicenter studies will hopefully go some way toward clarifying its exact role. Plus, close follow-up is essential for detection of early salvageable local or nodal recurrences irrespective to the choice of SNB observation or prophylactic neck dissection treatment of the N0 neck.

For those patients who can be followed-up closely, both elective neck dissection and excision of the primary lesion with SNB, without subsequent neck dissection have similar treatment results. The advantages and disadvantages of both observation and elective neck dissection should be clearly explained to the patient, and the treatment decision should be judged individually with the patient.

\section{Conclusions}

The rate of neck recurrence after SNB without neck dissection was not statistically significant compared to END in early stage HNSCC of the oral cavity in a matched pair analysis.

However, there are increasing scientific evidences that SNB can be used as a staging method, and the outcomes of this manuscript are consistent with that published literature.

\section{Acknowledgments}

The authors would like to thank all the patients and their families. They also wish to thank the ENT services and those who have contributed directly and indirectly to the realization of this study. Special thanks to ENT residents and the Head and Neck team of our ENT service.

\section{References}

1. Shah JP, Andersen PE (1994) The impact of patterns of nodal metastasis on modifications of neck dissection. Ann Surg Oncol 1: 521-532.

2. Rinaldo A, Ferlito A, Silver CE (2008) Early history of neck dissection. Eur Arch Otorhinolaryngol 265:1535-1538.

3. Byers RM (1985) Modified neck dissection: a study of 967 cases from 1970 to 1980. Am J Surg 150: 414-421.

4. Shah JP (1990) Patterns of cervical lymph node metastasis from squamous cell carcinoma of the upper aerodigestive tract. Am J Surg 160: 405-409.

5. Kowalski LP, Magrin J, Waksman G, Santo GF, Lopes ME, et al. (1993) Supraomohyoid neck dissection in the treatment of head and neck tumors: survival results in 212 cases. Arch Otolaryngol Head Neck Surg 119: 958963.

6. Atula TS, Grénman R, Varpula MJ, Kurki TJI, Klemi P (1996) Palpation, ultrasound, and ultrasound guided fine-needle aspiration cytology in the assessment of cervical lymph node status in head and neck cancer patients. Head Neck 18: 545-551.

7. Braams JW, Pruim J, Freling NJ, Nikkels PG, Roodenburg JL, et al. (1995) Detection of lymph node metastases of squamous cell cancer of the head and neck with FDG-PET and MRI. J Nucl Med 36: 211-216.

8. Merrit RM, Willians MF, James TH, Porubsky ES (1997) Detection of cervical metastasis. A meta-analysis comparing computed tomography with physical examination. Arch Otolaryngol Head Neck Surg 123: 149-152.

9. Woolgar JA, Beirne JC, Vaughan ED, Lewis-Jones HG, Scott J, et al. (1995) Correlation of histopathologic findings with clinical and radiologic assessments of cervicallymph node metastases in oral cancer. Int $\mathrm{J}$ Oral Maxillofac Surg 24: 30-37

10. Ambrosch P, Kron M, Fischer G, Brinck U (1995) Micro metastases in carcinoma of the upper aerodigestive tract: detection, risk of metastasizing, and prognostic value of depth of invasion. Head Neck 17: 473-479.

11. Mohit-Tarabatai MA, Sobel HJ, Rush BF, Mashberg A (1986) Relation of thickness of floor of mouth stage I and II cancers to regional metastasis. Am J Surg 152: 351-353.

12. Spiro RH, Huvos AG, Wong GY, Spiro JD (1986) Predictive value of tumor thickness in squamous cell carcinoma confined to tongue and floor of the mouth. Am J Surg 1152: 345-350.

13. Werner JA, Dunne AA, Ramaswamy A, Dalchow C, Behr T, et al. (2004) The sentinel node concept in head and neck cancer: solution for the controversies in the NO neck? Head Neck 26: 603-611.

14. Alkureishi LW, Ross GL, Shoaib T, Soutar DS, Robertson AG, et al. (2010) Sentinel node biopsy in head and neck squamous cell cancer: 5-year followup of a European multicenter trial. Ann Surg Oncol 17: 2459-2464. 
15. Fakih AR, Rao RS, Borges AM, Patel AR (1989) Elective versus therapeutic neck dissection in early carcinoma of the oral tongue. Am J Surg 158: 309313

16. Vandenbrouck C, Sancho-Garnier H, Chassagne D, Saravane D, Cachin $Y$ et al. (1980) Elective versus therapeutic radical neck dissection in epidermoid carcinoma of the oral cavity-results of a randomized clinical trial. Cancer 46 : 386-390.

17. Yuen AP, Ho CM, Chow TL, Tang LC, Cheung WY, et al. (2009) Prospective randomized study of selective neck dissection versus observation for N0 neck of early tonguecarcinoma. Head Neck 31: 765-772.

18. Stoeckli SJ, Pfaltz M, Ross GL (2005) The second international conference on sentinel node biopsy in mucosal head and neck cancer. Ann Surg Oncol 12: 919-924.

19. Devaney KO, Rinaldo A, Rodrigo JP (2006) Sentinel node biopsy and head and neck tumors-where do we stand today? Head Neck 28: 1122-1131.

20. Paleri V, Rees G, Arullendran P, Shoaib T, Krishman S (2005) Sentinel node biopsy in squamous cell cancer of the oral cavity and oral pharynx: a diagnostic meta-analysis. Head Neck 27: 739-747.

21. Tschopp L, Nuyens M, Stauffer E, Krause T, Zbären P (2005) The value of frozen section analysis of the sentinel lymph node in clinically NO squamous cell carcinoma of the oral cavity and oropharynx. Otolaryngol Head Neck Surg 132: 99-102.

22. Kovács AF, Landes CA, Hamscho N, Risse JH, Berner U, et al. (2005) Sentinel node biopsy as staging tool in a multimodality treatment approach to cancer of oral cavity and the oropharynx. Otolaryngol Head Neck Surg 132: 570-576.

23. Spiro RH, Morgan GJ, Strong EW, Shah JP (1996) Supraomohyoid neck dissection. Am J Surg 172: 650-653.

24. Strong EW (1969) Preoperative radiation and radical neck dissection. Surg Clin North Am 49: 271-276.

25. Edge SB, Byrd DR, Compton CC, Fritz AG, Greene FL, et al. Head and Neck. In: Edge SB, Byrd DR, Compton CC, Fritz AG, Greene FL, Trotti A (ed)
Cancer Staging Handbook from the AJCC Cancer Staging Manual, 7th edn. Springer, New York 21-100.

26. Shoaib T, Soutar DS, MacDonald DG, Camilleri IG, Dunaway DJ, et al. (2001) The accuracy of head and neck carcinoma sentinel lymph node biopsy in the clinically N0 neck. Cancer 91: 2077-2083.

27. Stoeckli SJ, Alkureishi LW, Ross GL (2009) Sentinel node biopsy for early oral and oropharyngeal squamous cell carcinoma. Eur Arch Otorhinolaryngol 266: 787-793.

28. Civantos FJ, Stoeckli SJ, Takes RP, et al. (2010) What is the role of sentinel lymph node biopsy in the management of oral cancer in 2010? Eur Arch Otorhinolaryngol 267: 839-844.

29. Civantos FJ, Zitsch RP, Schuller DE, Agrawal A, Smith RB, et al. (2010) Sentinel Lymph Node Biopsy Accurately Stages the Regional Lymph Nodes for T1-T2 Oral Squamous Cell Carcinomas: Results of a Prospective MultiInstitutional Trial. J Clin Oncol 28: 1395-1400.

30. Murer K, Huber GF, Haile SR, Stoeckli SJ (2011) Comparison of morbidity between sentinel node biopsy and elective neck dissection for treatment of the NO neck in patients with oral squamous cell carcinoma. Head Neck 33: 1260-1264.

31. Taylor RJ, Wahl RL, Sharma PK, Bradford CR, Terrell JE, et al. (2001) Sentinel node localization in oral cavity and oropharynx squamous cell cancer. Arch Otolaryngol Head Neck Surg 127: 970-974.

32. Ross GL (2008) On Behalf of the Sentinel European Node Trial (SENT) Organizing Committee. Sentinel node biopsy for squamous cell carcinoma of the oral cavity: preliminary results of the SENT trial. Presented at the annual meeting of the American Head and Neck Society (AHNS), San Francisco, CA.

33. Civantos F, Zitsch R, Bared A (2007) Sentinel node biopsy in oral squamous cell carcinoma. J Surg Oncol 96: 330-336.

34. Iype EM, Sebastian P, Mathew A, Balagopal PG, Varghese BT, et al. (2008) The role of selective neck dissection (I-III) in the treatment of node negative (N0) neck in oral cancer. Oral Oncol 44: 1134-1138.

Copyright: (c) 2016 de Carvalho GM, et al. This is an open-access article distributed under the terms of the Creative Commons Attribution License, which permits unrestricted use, distribution, and reproduction in any medium, provided the original author and source are credited. 